\title{
DYNAMICAL INVARIANTS FOR VARIABLE QUADRATIC HAMILTONIANS
}

\author{
SERGEI K. SUSLOV
}

\begin{abstract}
We consider linear and quadratic integrals of motion for general variable quadratic Hamiltonians. Fundamental relations between the eigenvalue problem for linear dynamical invariants and solutions of the corresponding Cauchy initial value problem for the time-dependent Schrödinger equation are emphasized. An eigenfunction expansion of the solution of the initial value problem is also found. A nonlinear superposition principle for generalized Ermakov systems is established as a result of decomposition of the general quadratic invariant in terms of the linear ones.
\end{abstract}

\section{An Introduction}

Quantum systems with variable quadratic Hamiltonians are called the generalized harmonic oscillators (see, for example, [2], [14, [15], 28], [39], [53], 84, 85] and references therein). They have attracted substantial attention over the years in view of their great importance in many advanced quantum problems. Examples include coherent states and uncertainty relations [53, [54, 55, 57, [33], Berry's phase [2], 3], [28], [39], [62], asymptotic and numerical methods [36], [58], [61], 63], quantization of mechanical systems [22], 223], 24], 25], [34, [35] and Hamiltonian cosmology [4], [26], 27], 29], charged particle traps [52] and motion in uniform magnetic fields [6], [10, [15], 37], [43], [44, [48], [55], molecular spectroscopy [16], [53] and polyatomic molecules in varying external fields, crystals through which an electron is passing and exciting the oscillator modes, and other interactions of the modes with external fields [25]. Quadratic Hamiltonians have particular applications in quantum electrodynamics because the electromagnetic field can be represented as a set of forced harmonic oscillators [5], 15, [25], 60] and [73]. Nonlinear oscillators play a central role in the novel theory of Bose-Einstein condensation [11]. From a general point of view, the dynamics of gases of cooled atoms in a magnetic trap at very low temperatures can be described by an effective equation for the condensate wave function known as the Gross-Pitaevskii (or nonlinear Schrödinger) equation [30], 31], 32] and [68.

In this Letter we consider the one-dimensional time-dependent Schrödinger equation

$$
i \frac{\partial \psi}{\partial t}=H \psi
$$

with general variable quadratic Hamiltonians of the form

$$
H=a(t) p^{2}+b(t) x^{2}+c(t) p x+d(t) x p, \quad p=-i \frac{\partial}{\partial x},
$$

where $a(t), b(t), c(t)$, and $d(t)$ are real-valued functions of time, $t$, only (see, for example, [6], [7, [8], [9], [14], 23], [24], [25], [38], [50], [59], [76], [77], [78], [79], [84], and [85] for a general approach

Date: October 29, 2018.

1991 Mathematics Subject Classification. Primary 81Q05, 35C05. Secondary 42A38.

Key words and phrases. The time-dependent Schrödinger equation, Cauchy initial value problem, Green function, propagator, dynamical symmetry, quantum integrals of motion and dynamical invariants, Ermakov's systems. 
and some elementary solutions). The corresponding Green function, or Feynman's propagator, can be found as follows [6], [78]:

$$
\psi=G(x, y, t)=\frac{1}{\sqrt{2 \pi i \mu_{0}(t)}} e^{i\left(\alpha_{0}(t) x^{2}+\beta_{0}(t) x y+\gamma_{0}(t) y^{2}\right)},
$$

where

$$
\begin{aligned}
& \alpha_{0}(t)=\frac{1}{4 a(t)} \frac{\mu_{0}^{\prime}(t)}{\mu_{0}(t)}-\frac{c(t)}{2 a(t)}, \\
& \beta_{0}(t)=-\frac{\lambda(t)}{\mu_{0}(t)}, \quad \lambda(t)=\exp \left(\int_{0}^{t}(c(s)-d(s)) d s\right), \\
& \gamma_{0}(t)=\frac{a(t) \lambda^{2}(t)}{\mu_{0}(t) \mu_{0}^{\prime}(t)}+\frac{c(0)}{2 a(0)}-4 \int_{0}^{t} \frac{a(s) \sigma(s) \lambda^{2}(s)}{\left(\mu_{0}^{\prime}(s)\right)^{2}} d s,
\end{aligned}
$$

and the function $\mu_{0}(t)$ satisfies the characteristic equation

$$
\mu^{\prime \prime}-\tau(t) \mu^{\prime}+4 \sigma(t) \mu=0
$$

with

$$
\tau(t)=\frac{a^{\prime}}{a}+2 c-2 d, \quad \sigma(t)=a b-c d+\frac{c}{2}\left(\frac{a^{\prime}}{a}-\frac{c^{\prime}}{c}\right)
$$

subject to the initial data

$$
\mu_{0}(0)=0, \quad \mu_{0}^{\prime}(0)=2 a(0) \neq 0 .
$$

(More details can be found in Refs. [6] and [78].) Then by the superposition principle the solution of the Cauchy initial value problem can be presented in an integral form

$$
\psi(x, t)=\int_{-\infty}^{\infty} G(x, y, t) \varphi(y) d y, \quad \lim _{t \rightarrow 0^{+}} \psi(x, t)=\varphi(x)
$$

for a suitable initial function $\varphi$ on $\mathbb{R}$ (a rigorous proof is given in [78] and uniqueness is analyzed in [8]; another forms of solution are provided by (4.20) and (7.11) $)$.

A detailed review on dynamical symmetries and quantum integrals of motion for the timedependent Schrödinger equation can be found in [15] and [53] (see also an extensive list of references therein). In this Letter, which is a continuation of the recent paper [8], a natural connection between the linear and quadratic integrals of motion for general variable quadratic Hamiltonians is established. As a result a nonlinear superposition principle for the corresponding Ermakov systems, known as Pinney's solution, is obtained and generalized. We pay also special attention to fundamental relations between the linear dynamical invariants of Dodonov, Malkin, Man'ko and Trifonov and solutions of the Cauchy initial value problem (see original works [14], [15], [53], [56]). In addition this initial value problem is explicitly solved in terms of the quadratic invariant eigenfunction expansion, which seems to be missing in the available literature in general.

The Letter is organized as follows. We start from the standard definitions of the dynamical symmetry and quantum integrals of motion, introducing also some elementary tools, in the next two sections. Then in Section 4 we describe all linear dynamical invariants for variable quadratic Hamiltonians and determine their actions on the solutions of the corresponding time-dependent Schrödinger equation. In Section 5 all quadratic quantum integrals of motion are characterized in terms of solutions of the generalized Ermakov equation and a detailed proof is given. Their 
decomposition into products of the linear invariants is derived in Section 6 and explicit actions on the solutions are established in Section 7. The last section deals with a related nonlinear superposition principle for Ermakov's equations and some computational details are provided in Appendices A and B.

\section{Dynamical Symmetry}

In this Letter we elaborate on the following property.

Lemma 1. If

$$
i \frac{\partial \psi}{\partial t}=H \psi, \quad i \frac{\partial O}{\partial t}+O H-H^{\dagger} O=0
$$

then the function $\psi_{1}=O \psi$ satisfies the time-dependent Schrödinger equation

$$
i \frac{\partial \psi_{1}}{\partial t}=H^{\dagger} \psi_{1}
$$

where $H^{\dagger}$ is the adjoint of the Hamiltonian $H$.

When $H=H^{\dagger}$, this property is taken as a definition of the dynamical symmetry of the timedependent Schrödinger equation (1.1) (see, for example, [15, [48, [53] and references therein). At the same time one has to deal with non-self-adjoint Hamiltonians in the theory of dissipative quantum systems (see, for example, [7, [13], [35], 81, [82 and references therein) or when using separation of variables in an accelerating frame of reference for a charged particle moving in an uniform variable magnetic field [6].

Proof. Partial differentiation

$$
\begin{aligned}
i \frac{\partial \psi_{1}}{\partial t} & =i \frac{\partial}{\partial t}(O \psi)=i \frac{\partial O}{\partial t} \psi+i O \frac{\partial \psi}{\partial t} \\
& =\left(H^{\dagger} O-O H\right) \psi+O H \psi=H^{\dagger} \psi_{1}
\end{aligned}
$$

provides a direct proof.

Definition of the dynamical symmetry is usually given in terms of solutions of the same equation. A simple modification helps with the non-self-adjoint quadratic Hamiltonians.

Lemma 2. The wave functions $\psi$ and $\chi$, related by

$$
\psi=\left(e^{-\int_{0}^{t}(c-d) d s} O\right) \chi
$$

are solutions of the same Schrödinger equation (1.1)-(1.2), if the operator $O$ satisfies hypothesis of Lemma 1.

Proof. The simplest dynamical invariant, or an operator with the property (2.1), is given by

$$
O_{0}=O_{0}(c, d)=e^{\int_{0}^{t}(c-d) d s} I
$$

where $I=i d$ is the identity operator. (More details are provided in Section 4.) Apply Lemma 1 twice in the following order

$$
\psi=O_{0}(d, c)(O \chi)
$$

and use $\left(H^{\dagger}\right)^{\dagger}=H$ to complete the proof. 
Examples occur throughout the Letter.

\section{Differentiation of Operators and Dynamical Invariants}

Following Lemma 1 we define the time derivative of an operator $O$ as follows

$$
\frac{d O}{d t}=\frac{\partial O}{\partial t}+\frac{1}{i}\left(O H-H^{\dagger} O\right)
$$

where $H^{\dagger}$ is the adjoint of the Hamiltonian operator $H$. (This formula is a simple extension of the well-known expression [12], [37], 60], [73] to the case of a non-self-adjoint Hamiltonian [7].) By definition

$$
\frac{d O}{d t}=\frac{\partial O}{\partial t}+\frac{1}{i}\left(O H-H^{\dagger} O\right)=0
$$

for any dynamical invariant. (In what follows we assume that the invariant $O$ does not involve time differentiation.)

This derivative is a linear operator

$$
\frac{d}{d t}\left(c_{1} O_{1}+c_{2} O_{2}\right)=c_{1} \frac{d O_{1}}{d t}+c_{2} \frac{d O_{2}}{d t}
$$

and the product rule takes the form

$$
\begin{aligned}
\frac{d}{d t}\left(O_{1} O_{2}\right) & =\frac{\partial\left(O_{1} O_{2}\right)}{\partial t}+\frac{1}{i}\left(\left(O_{1} O_{2}\right) H-H^{\dagger}\left(O_{1} O_{2}\right)\right) \\
& =\frac{d O_{1}}{d t} O_{2}+O_{1} \frac{d O_{2}}{d t}+i O_{1}\left(H-H^{\dagger}\right) O_{2} .
\end{aligned}
$$

For the general quadratic Hamiltonian, (1.2), one gets

$$
\frac{d}{d t}\left(O_{1} O_{2}\right)=\frac{d O_{1}}{d t} O_{2}+O_{1} \frac{d O_{2}}{d t}+(c-d) O_{1} O_{2}
$$

and by definition (3.1)

$$
\begin{aligned}
\frac{d}{d t}\left(e^{\alpha \int_{0}^{t}(c-d) d s} O_{1} O_{2}\right)= & e^{\alpha \int_{0}^{t}(c-d) d s}\left(\frac{d O_{1}}{d t} O_{2}+O_{1} \frac{d O_{2}}{d t}\right) \\
& +(\alpha+1)(c-d) e^{\alpha \int_{0}^{t}(c-d) d s} O_{1} O_{2} .
\end{aligned}
$$

If $\alpha=-1$, we finally obtain

$$
\frac{d}{d t}\left(e^{-\int_{0}^{t}(c-d) d s} O_{1} O_{2}\right)=e^{-\int_{0}^{t}(c-d) d s}\left(\frac{d O_{1}}{d t} O_{2}+O_{1} \frac{d O_{2}}{d t}\right) .
$$

This implies that, if the operators $O_{1}$ and $O_{2}$ are dynamical invariants, namely,

$$
\begin{aligned}
& \frac{d O_{1}}{d t}=\frac{\partial O_{1}}{\partial t}+\frac{1}{i}\left(O_{1} H-H^{\dagger} O_{1}\right)=0 \\
& \frac{d O_{2}}{d t}=\frac{\partial O_{2}}{\partial t}+\frac{1}{i}\left(O_{2} H-H^{\dagger} O_{2}\right)=0
\end{aligned}
$$

then their modified product,

$$
E=e^{-\int_{0}^{t}(c-d) d s} O_{1} O_{2}
$$


is also a dynamical invariant:

$$
\frac{d E}{d t}=\frac{\partial E}{\partial t}+\frac{1}{i}\left(E H-H^{\dagger} E\right)=0 .
$$

In Section 6 this property allows us to describe connections between linear and quadratic dynamical invariants of the time-dependent Hamiltonian (1.2).

\section{Linear Integrals of Motion}

All invariants of the form

$$
P=A(t) p+B(t) x+C(t), \quad \frac{d P}{d t}=0,
$$

(we call them the Dodonov-Malkin-Man'ko-Trifonov invariants; see, for example, [14], [15], [53, and [56] and references therein) for the general variable quadratic Hamiltonian (1.2) can be found in the following fashion. Use of the differentiation formula (3.1) results in the system [8]:

$$
\begin{aligned}
& A^{\prime}=2 c(t) A-2 a(t) B, \\
& B^{\prime}=2 b(t) A-2 d(t) B, \\
& C^{\prime}=(c(t)-d(t)) C .
\end{aligned}
$$

The last equation is explicitly integrated and elimination of $B$ and $A$ from (4.2) and (4.3), respectively, gives the second-order equations:

$$
\begin{aligned}
& A^{\prime \prime}-\left(\frac{a^{\prime}}{a}+2 c-2 d\right) A^{\prime}+4\left(a b-c d+\frac{c}{2}\left(\frac{a^{\prime}}{a}-\frac{c^{\prime}}{c}\right)\right) A=0 \\
& B^{\prime \prime}-\left(\frac{b^{\prime}}{b}+2 c-2 d\right) B^{\prime}+4\left(a b-c d-\frac{d}{2}\left(\frac{b^{\prime}}{b}-\frac{d^{\prime}}{d}\right)\right) B=0 .
\end{aligned}
$$

The first is simply the characteristic equation (1.7)-(1.8). (It also coincides with the Ehrenfest theorem $([8]$, [17]) when $c \leftrightarrow d$.)

Thus all linear quantum invariants are given by

$$
P=A(t) p+\frac{2 c(t) A(t)-A^{\prime}(t)}{2 a(t)} x+C_{0} \exp \left(\int_{0}^{t}(c(s)-d(s)) d s\right),
$$

where $A(t)$ is a general solution of equation (4.5) depending upon two parameters and $C_{0}$ is the third constant. Study of spectra of the linear dynamical invariants allows one to solve the Cauchy initial value problem ([14], [15], [53] and [56]).

Theorem 1. (Eigenvalue Problem for the Linear Invariants) If

$$
P(t)=\mu(t) p+\frac{2 c(t) \mu(t)-\mu^{\prime}(t)}{2 a(t)} x,
$$

then for any solution $A=\mu(t)$ of the characteristic equation (1.7)-(1.8) we have

$$
P(t) K(x, y, t)=\beta(0) \mu(0) \lambda(t) \text { y } K(x, y, t) .
$$

The eigenfunctions are bounded solutions of the time-dependent Schrödinger equation (1.1) given by

$$
K(x, y, t)=\frac{1}{\sqrt{2 \pi \mu(t)}} e^{i\left(\alpha(t) x^{2}+\beta(t) x y+\gamma(t) y^{2}\right)},
$$




$$
\begin{aligned}
\text { where } \lambda(t)=\exp \left(\int_{0}^{t}(c(s)-d(s))\right. & d s) \text { and } \\
\mu(t) & =2 \mu(0) \mu_{0}(t)\left(\alpha(0)+\gamma_{0}(t)\right), \\
\alpha(t) & =\frac{1}{4 a(t)} \frac{\mu^{\prime}(t)}{\mu(t)}-\frac{c(t)}{2 a(t)} \\
& =\alpha_{0}(t)-\frac{\beta_{0}^{2}(t)}{4\left(\alpha(0)+\gamma_{0}(t)\right)}, \\
\beta(t) & =-\frac{\beta(0) \beta_{0}(t)}{2\left(\alpha(0)+\gamma_{0}(t)\right)} \\
& =\frac{\beta(0) \mu(0)}{\mu(t)} \lambda(t) \\
\gamma(t) & =\gamma(0)-\frac{\beta^{2}(0)}{4\left(\alpha(0)+\gamma_{0}(t)\right)} .
\end{aligned}
$$

(When $\mu=\mu_{0}$ with $\mu_{0}(0)=0$ and $\mu_{0}^{\prime}(0)=2 a(0) \neq 0$, we obtain the Green function (1.3) $-(1.6)$ up to a simple factor.)

Proof. The required solution (4.10)-(4.14) has been already constructed in [78] and one has to verify formula (4.9) only. We have

$$
\begin{aligned}
P K(x, y, t) & =(A p+B x) K(x, y, t) \\
& =((2 \alpha A+B) x+\beta A y) K(x, y, t)
\end{aligned}
$$

where

$$
\begin{aligned}
2 \alpha A+B & =\left(\frac{1}{2 a} \frac{\mu^{\prime}}{\mu}-\frac{c}{a}\right) A+\frac{2 c A-A^{\prime}}{2 a} \\
& =\frac{A}{2 a}\left(\frac{\mu^{\prime}}{\mu}-\frac{A^{\prime}}{A}\right)=0
\end{aligned}
$$

provided $\mu=A$. Then $\beta A=\beta(0) \mu(0) \lambda$ and the proof is complete.

Our theorem can be thought as a natural extension to the case of non-self-adjoint variable quadratic Hamiltonians (1.2) of a familiar relation between the Green function and linear dynamical invariants established in [14], [15], [53] and [56]. The time-dependent factor in the eigenvalue (4.9) corresponds to the statement of Lemma 2.

In this Letter we are interested, among other things, in a direct verification of Lemma 1 for linear and quadratic dynamical invariants. For the general Dodonov-Malkin-Man'ko-Trifonov invariant (4.7), without any loss of generality, one can separately consider two cases, say, when $A(t) \equiv 0$ with $C_{0}=1$ and when $C_{0}=0$. If

$$
\psi_{1}=\psi e^{\int_{0}^{t}(c-d) d s}
$$

then

$$
\begin{aligned}
i \frac{\partial \psi_{1}}{\partial t}= & i \frac{\partial \psi}{\partial t} e^{\int_{0}^{t}(c-d) d s}+i(c-d) \psi_{1} \\
= & \left(a p^{2}+b x^{2}+c p x+d x p\right) \psi_{1} \\
& +(c-d)(x p-p x) \psi_{1}=H^{\dagger} \psi_{1}
\end{aligned}
$$


which takes care of the first case (we have verified once again the statement of Lemma 1 for the simplest invariant (2.5) ).

In the second case, $C_{0}=0$, we follow Theorem 1 and take a solution $A=\mu(t)$ of (4.5) and (1.7), which does not have to satisfy the initial conditions of the Green function (1.9). Then by the superposition principle solution of the corresponding Cauchy initial values problem is given by the integral operator [78]

$$
\psi(x, t)=\int_{-\infty}^{\infty} K(x, y, t) \chi(y) d y, \quad \psi(x, 0)=\int_{-\infty}^{\infty} K(x, y, 0) \chi(y) d y
$$

with the kernel (4.10)-(4.14). Thus

$$
\psi_{1}(x, t)=P \psi(x, t)=\int_{-\infty}^{\infty}(P K(x, y, t)) \chi(y) d y
$$

(we freely interchange differentiation and integration in this Letter; it can be justified for certain classes of solutions ([49], [66], [68], [83])). By choosing $\beta(0) \mu(0)=1$ in (4.9) we obtain

$$
\psi_{1}(x, t)=P \psi(x, t)=e^{\int_{0}^{t}(c-d) d s} \int_{-\infty}^{\infty} K(x, y, t)(y \chi(y)) d y,
$$

where the second factor obviously satisfies the Schrödinger equation (1.11) (see [78] for details). Repeating the first step one completes the proof. Eq. (4.22) provides a spectral decomposion ([70], [71]) of the linear invariant $P$, which has a continuous spectrum.

Our consideration shows how Lemma 1 works for the general Dodonov-Malkin-Man'ko-Trifonov invariant - application of this invariant to a given solution of the corresponding Cauchy initial value problem simply produces another solution with the following initial data:

$$
\begin{aligned}
\psi_{1}(x, 0) & =P(0) \psi(x, 0) \\
& =\int_{-\infty}^{\infty} K(x, y, 0)(y \chi(y)) d y .
\end{aligned}
$$

The reader can easily connect initial conditions of two solutions (4.20) and (4.22) with the help of an analog of the Fourier transform (see also Ref. [77] for a formal inversion operator in general).

\section{Quadratic Dynamical InVARIANTS}

All quantum quadratic integrals of motion are given by

$$
E=A(t) p^{2}+B(t) x^{2}+C(t)(p x+x p), \quad \frac{d E}{d t}=0,
$$

and can be found as follows [8].

Lemma 3. The quadratic dynamical invariants of the Hamiltonian (1.2) have the form

$$
\begin{aligned}
E(t)= & {\left[\left(\kappa p+\frac{(c+d) \kappa-\kappa^{\prime}}{2 a} x\right)^{2}+\frac{C_{0}}{\kappa^{2}} x^{2}\right] } \\
& \times \exp \left(\int_{0}^{t}(c-d) d s\right)
\end{aligned}
$$


where $C_{0}$ is a constant and function $\kappa(t)$ is a solution of the following nonlinear auxiliary equation:

$$
\kappa^{\prime \prime}-\frac{a^{\prime}}{a} \kappa^{\prime}+\left[4 a b+\left(\frac{a^{\prime}}{a}-c-d\right)(c+d)-c^{\prime}-d^{\prime}\right] \kappa=C_{0} \frac{(2 a)^{2}}{\kappa^{3}} .
$$

The structure of quadratic invariants is once again in perfect agreement with Lemma 2.

Proof. In a general form this result has been established in [8] (see also Refs. [48], [80] and [85] for important earlier articles). A somewhat different and more direct proof is presented here. It is sufficient to show that the corresponding linear system

$$
\begin{aligned}
A^{\prime}+4 a C-(3 c+d) A & =0 \\
B^{\prime}-4 b C+(c+3 d) B & =0 \\
C^{\prime}+2(a B-b A)-(c-d) C & =0
\end{aligned}
$$

has the following general solution

$$
\begin{aligned}
A(t) & =\kappa^{2} \exp \left(\int_{0}^{t}(c-d) d s\right) \\
B(t) & =\left[\left(\frac{\kappa^{\prime}-(c+d) \kappa}{2 a}\right)^{2}+\frac{C_{0}}{\kappa^{2}}\right] \exp \left(\int_{0}^{t}(c-d) d s\right), \\
C(t) & =\kappa \frac{(c+d) \kappa-\kappa^{\prime}}{2 a} \exp \left(\int_{0}^{t}(c-d) d s\right),
\end{aligned}
$$

where $C_{0}$ is a constant and the function $\kappa(t)$ satisfies the nonlinear auxiliary equation (5.3).

Indeed the substitution

$$
A(t)=\widetilde{A}(t) \lambda(t), \quad B(t)=\widetilde{B}(t) \lambda(t), \quad C(t)=\widetilde{C}(t) \lambda(t),
$$

where $\lambda(t)=\exp \left(\int_{0}^{t}(c-d) d s\right)$, transforms the original system into a more convenient form

$$
\begin{aligned}
\widetilde{A}^{\prime}+4 a \widetilde{C}-2(c+d) \widetilde{A} & =0 \\
\widetilde{B}^{\prime}-4 b \widetilde{C}+2(c+d) \widetilde{B} & =0 \\
\widetilde{C}^{\prime}+2(a \widetilde{B}-b \widetilde{A}) & =0 .
\end{aligned}
$$

Letting

$$
\widetilde{A}=\kappa^{2} \quad \text { and } \quad \widetilde{A}^{\prime}=2 \kappa \kappa^{\prime}
$$

in the first equation (5.11), we obtain

$$
\begin{aligned}
\widetilde{C} & =\frac{\kappa}{2 a}\left((c+d) \kappa-\kappa^{\prime}\right) \\
& =-e^{\int(c+d) d t} \frac{\kappa}{2 a} \frac{d}{d t}\left(\kappa e^{-\int(c+d) d t}\right) .
\end{aligned}
$$

Then from the third equation (5.13):

$$
\widetilde{B}=\frac{b}{a} \kappa^{2}+\frac{1}{2 a}\left[\kappa \frac{\kappa^{\prime}-(c+d) \kappa}{2 a}\right]^{\prime}
$$




$$
=\frac{b}{a} \kappa^{2}+\frac{1}{2 a} \frac{d}{d t}\left[e^{\int(c+d) d t} \frac{\kappa}{2 a} \frac{d}{d t}\left(\kappa e^{-\int(c+d) d t}\right)\right]
$$

and substitution of (5.15)-(5.16) into (5.12) gives

$$
k \frac{d}{d t}\left[4 a b k^{2} \mu^{2}+k \frac{d}{d t}\left(\mu\left(k \frac{d \mu}{d t}\right)\right)\right]+8 a b k^{2} \mu\left(k \frac{d \mu}{d t}\right)=0
$$

in the following temporary notations

$$
\mu=\kappa e^{-\int(c+d) d t}, \quad k=\frac{1}{2 a} e^{2 \int(c+d) d t} .
$$

Using

$$
\mu(t)=y(\tau), \quad k \frac{d \mu}{d t}=\frac{d y}{d \tau}, \quad q=4 a b k^{2}=\frac{b}{a} e^{4 \int(c+d) d t},
$$

one gets

$$
\frac{d}{d \tau}\left[q y^{2}+\frac{d}{d \tau}\left(y \frac{d y}{d \tau}\right)\right]+2 q y \frac{d y}{d \tau}=0
$$

or

$$
y\left(y^{\prime \prime}+q y\right)^{\prime}+3 y^{\prime}\left(y^{\prime \prime}+q y\right)=0
$$

(see also Ref. [48] for an important special case). By an integrating factor:

$$
\frac{d}{d \tau}\left[y^{3}\left(y^{\prime \prime}+q y\right)\right]=0, \quad y^{\prime \prime}+q y=\frac{C_{0}}{y^{3}},
$$

and a back substitution with the help of

$$
\frac{d^{2} y}{d \tau^{2}}=\frac{e^{3 \int(c+d) d t}}{(2 a)^{2}}\left[\kappa^{\prime \prime}-\frac{a^{\prime}}{a} \kappa^{\prime}+\left(\left(\frac{a^{\prime}}{a}-c-d\right)(c+d)-c^{\prime}-d^{\prime}\right) \kappa\right]
$$

results in the required first integral of the system:

$$
\frac{d}{d t}\left[\frac{\kappa^{3}}{(2 a)^{2}}\left(\kappa^{\prime \prime}-\frac{a^{\prime}}{a} \kappa^{\prime}+\left(4 a b+\left(\frac{a^{\prime}}{a}-c-d\right)(c+d)-c^{\prime}-d^{\prime}\right) \kappa\right)\right]=0,
$$

which gives our auxiliary equation (5.3).

The last term in (5.16) can be transformed as follows

$$
\begin{aligned}
\frac{1}{2 a} \frac{d}{d t} & {\left[e^{\int(c+d) d t} \frac{\kappa}{2 a} \frac{d}{d t}\left(\kappa e^{-\int(c+d) d t}\right)\right] } \\
& =e^{-2 \int(c+d) d t} \frac{d}{d \tau}\left(y \frac{d y}{d \tau}\right)=e^{-2 \int(c+d) d t}\left(y y^{\prime \prime}+\left(y^{\prime}\right)^{2}\right) \\
& =e^{-2 \int(c+d) d t}\left[\left(\frac{d y}{d \tau}\right)^{2}-q y^{2}+\frac{C_{0}}{y^{2}}\right] \\
& =\left(\frac{\kappa^{\prime}-(c+d) \kappa}{2 a}\right)^{2}-\frac{b}{a} \kappa^{2}+\frac{C_{0}}{\kappa^{2}}
\end{aligned}
$$

in view of (5.18)-(5.19) and (5.22). We have also utilized a convenient identity

$$
\frac{d y}{d \tau}=\frac{1}{2 a}\left(\frac{d \kappa}{d t}-(c+d) \kappa\right) e^{\int(c+d) d t} .
$$


Thus

$$
\widetilde{B}=\left(\frac{\kappa^{\prime}-(c+d) \kappa}{2 a}\right)^{2}+\frac{C_{0}}{\kappa^{2}}
$$

and the proof is complete.

The case $a=1 / 2, b=\omega^{2}(t) / 2$ and $c=d=0$ corresponds to the familiar Ermakov-LewisRiesenfeld invariant [21], [43], [44], 45], [48]; see also Refs. [46] and [47]. (The corresponding classical invariant in general is discussed in Refs. [80] and [85].) Many examples of completely integrable generalized harmonic oscillators and their integrals of motion are given in Ref. [8].

For a positive constant, $C_{0}>0$, the quantum dynamical invariant (5.2) can be presented in the standard harmonic oscillator form [7], 8], 70], [71]:

$$
E=\frac{\omega(t)}{2}\left(\widehat{a}(t) \widehat{a}^{\dagger}(t)+\widehat{a}^{\dagger}(t) \widehat{a}(t)\right)
$$

where

$$
\begin{gathered}
\omega(t)=\omega_{0} \exp \left(\int_{0}^{t}(c-d) d s\right), \quad \omega_{0}=2 \sqrt{C_{0}}>0, \\
\widehat{a}(t)=\left(\frac{\sqrt{\omega_{0}}}{2 \kappa}-i \frac{\kappa^{\prime}-(c+d) \kappa}{2 a \sqrt{\omega_{0}}}\right) x+\frac{\kappa}{\sqrt{\omega_{0}}} \frac{\partial}{\partial x} \\
\widehat{a}^{\dagger}(t)=\left(\frac{\sqrt{\omega_{0}}}{2 \kappa}+i \frac{\kappa^{\prime}-(c+d) \kappa}{2 a \sqrt{\omega_{0}}}\right) x-\frac{\kappa}{\sqrt{\omega_{0}}} \frac{\partial}{\partial x}
\end{gathered}
$$

and $\kappa$ is a real-valued solution of the nonlinear auxiliary equation (5.3). Here the time-dependent annihilation $\widehat{a}(t)$ and creation $\widehat{a}^{\dagger}(t)$ operators satisfy the canonical commutation relation:

$$
\widehat{a}(t) \widehat{a}^{\dagger}(t)-\widehat{a}^{\dagger}(t) \widehat{a}(t)=1 .
$$

The oscillator-type spectrum of the dynamical invariant $E$ can be obtained now in a standard way by using the Heisenberg-Weyl algebra of the rasing and lowering operators (a "second quantization" [48], the Fock states):

$$
\begin{gathered}
\widehat{a}(t) \Psi_{n}(x, t)=\sqrt{n} \Psi_{n-1}(x, t), \quad \widehat{a}^{\dagger}(t) \Psi_{n}(x, t)=\sqrt{n+1} \Psi_{n+1}(x, t), \\
E(t) \Psi_{n}(x, t)=\omega(t)\left(n+\frac{1}{2}\right) \Psi_{n}(x, t) .
\end{gathered}
$$

The corresponding orthonormal time-dependent eigenfunctions are given by

$$
\Psi_{n}(x, t)=\exp \left(i \frac{\kappa^{\prime}-(c+d) \kappa}{4 a \kappa} x^{2}\right) v_{n}(\xi)
$$

in terms of Hermite polynomials [64]:

$$
v_{n}=C_{n} e^{-\xi^{2} / 2} H_{n}(\xi), \quad \xi=\varepsilon x
$$

where

$$
\left|C_{n}\right|^{2}=\frac{\varepsilon}{\sqrt{\pi} 2^{n} n !}, \quad \varepsilon^{2}=\frac{\omega_{0}}{2 \kappa^{2}}=\frac{\sqrt{C_{0}}}{\kappa^{2}} .
$$

Their relation with the original Cauchy initial value problem is discussed in Section 7 (see Theorem 2). In addition the $n$-dimensional oscillator wave functions form a basis of the irreducible unitary representation of the Lie algebra of the noncompact group $S U(1,1)$ corresponding to the 
discrete positive series $\mathcal{D}_{+}^{j}$ (see [59], 64] and [75]). Operators (5.29)-(5.30) allow us to extend these group-theoretical properties to the general quadratic dynamical invariant (5.27).

When $C_{0}=0$, the dynamical invariant (5.2) is given as the square of a linear invariant up to a simple factor, which resembles the case of a free particle. If $C_{0}<0$, one deals with the Hamiltonian of a linear repulsive "oscillator".

\section{Relation Between Linear and Quadratic Invariants}

By Lemma 3 the operators $p^{2}, x^{2}$ and $p x+x p$ form a basis for all quadratic invariants. Here we take two linearly independent solutions, say $\mu_{1}=A_{1}$ and $\mu_{2}=A_{2}$, of equations (1.7) and (4.5) and consider the corresponding Dodonov-Malkin-Man'ko-Trifonov invariants (4.7):

$$
P_{1}=A_{1} p+B_{1} x, \quad P_{2}=A_{2} p+B_{2} x .
$$

Introducing the following quadratic invariants

$$
E_{1}=P_{1}^{2} e^{-\int_{0}^{t}(c-d) d s}, \quad E_{2}=P_{2}^{2} e^{-\int_{0}^{t}(c-d) d s}, \quad E_{3}=\left(P_{1} P_{2}+P_{2} P_{1}\right) e^{-\int_{0}^{t}(c-d) d s}
$$

with the help of (3.10) as another basis, one gets

$$
\begin{aligned}
E & =C_{1} E_{1}+C_{2} E_{2}+C_{3} E_{3} \\
& =\left[C_{1} P_{1}^{2}+C_{2} P_{2}^{2}+C_{3}\left(P_{1} P_{2}+P_{2} P_{1}\right)\right] \exp \left(-\int_{0}^{t}(c-d) d s\right)
\end{aligned}
$$

for some constants $C_{1}, C_{2}$ and $C_{3}$. As a result the following operator identity holds

$$
\begin{aligned}
& {\left[\left(\kappa p+\frac{(c+d) \kappa-\kappa^{\prime}}{2 a} x\right)^{2}+\frac{C_{0}}{\kappa^{2}} x^{2}\right] \exp \left(\int_{0}^{t}(c-d) d s\right)} \\
& =\left[C_{1}\left(A_{1} p+B_{1} x\right)^{2}+C_{2}\left(A_{2} p+B_{2} x\right)^{2}\right. \\
& \left.\quad+C_{3}\left(\left(A_{1} p+B_{1} x\right)\left(A_{2} p+B_{2} x\right)+\left(A_{2} p+B_{2} x\right)\left(A_{1} p+B_{1} x\right)\right)\right] \\
& \quad \times \exp \left(-\int_{0}^{t}(c-d) d s\right)
\end{aligned}
$$

where

Thus we obtain

$$
A_{1}=\mu_{1}, \quad B_{1}=\frac{2 c \mu_{1}-\mu_{1}^{\prime}}{2 a}, \quad A_{2}=\mu_{2}, \quad B_{2}=\frac{2 c \mu_{2}-\mu_{2}^{\prime}}{2 a}
$$

$$
\kappa^{2}=\left(C_{1} \mu_{1}^{2}+C_{2} \mu_{2}^{2}+2 C_{3} \mu_{1} \mu_{2}\right) \exp \left(-2 \int_{0}^{t}(c-d) d s\right)
$$

as a relation between solutions of the nonlinear auxiliary equation (5.3) and the linear characteristic equation (4.5). In addition the substitution

$$
\mu_{1}=\kappa_{1} \exp \left(\int_{0}^{t}(c-d) d s\right), \quad \mu_{2}=\kappa_{2} \exp \left(\int_{0}^{t}(c-d) d s\right)
$$

transforms the characteristic equation (4.5) into our auxiliary equation (5.3) with $C_{0}=0$. Finally a general solution of the nonlinear equation is given by the following "operator law of cosines":

$$
\kappa^{2}(t)=C_{1} \kappa_{1}^{2}(t)+C_{2} \kappa_{2}^{2}(t)+2 C_{3} \kappa_{1}(t) \kappa_{2}(t)
$$


in terms of two linearly independent solutions $\kappa_{1}$ and $\kappa_{2}$ of the homogeneous equation. The constant $C_{0}$ is related to the Wronskian of two linearly independent solutions $\kappa_{1}$ and $\kappa_{2}$ :

$$
C_{1} C_{2}-C_{3}^{2}=C_{0} \frac{(2 a)^{2}}{W^{2}\left(\kappa_{1}, \kappa_{2}\right)}, \quad W\left(\kappa_{1}, \kappa_{2}\right)=\kappa_{1} \kappa_{2}^{\prime}-\kappa_{1}^{\prime} \kappa_{2}
$$

(more details are given in Appendix A). This is a well-known nonlinear superposition property of the so-called Ermakov systems (see, for example, [10], [18], [21], 40], [41], [42], [45], [51], [65], [67], [69], [74] and references therein). Here we have obtained this "nonlinear superposition principle" (or Pinney's solution) in an operator form by multiplication and addition of the linear dynamical invariants together with an independent characterization of all quantum quadratic invariants, which seems to be missing, in general, in the available literature (see also [43] and [45] for an important classical case). An extension is given in the last section.

It is worth noting, in conclusion, that the linear invariants of Dodonov, Malkin, Man'ko and Trifonov ([14], [15], [53] and [56]) can be presented as follows

$$
\begin{aligned}
& P_{1}=\left(\kappa_{1} p+\frac{(c+d) \kappa_{1}-\kappa_{1}^{\prime}}{2 a} x\right) \exp \left(\int_{0}^{t}(c-d) d s\right), \\
& P_{2}=\left(\kappa_{2} p+\frac{(c+d) \kappa_{2}-\kappa_{2}^{\prime}}{2 a} x\right) \exp \left(\int_{0}^{t}(c-d) d s\right)
\end{aligned}
$$

in terms of two linearly independent solutions, $\kappa_{1}$ and $\kappa_{2}$, of the homogeneous equation (5.3) when $C_{0}=0$. Comparing these expressions with the form of the quadratic invariant (5.2) at $C_{0}=0$ (no "potential", a "free particle"), when the operator square is complete, one can treat the linear invariants as "operator square roots" [12] of the special quadratic invariants (see also Lemma 2 regarding a convenient common factor).

\section{Quadratic Invariants and Cauchy Initial Value Problem}

Our decomposition (6.3) of the quantum quadratic invariant in terms of products of the linear ones not only results in the Pinney solution (6.8)-(6.9) of the corresponding generalized Ermakov system (5.3) in a form of an "operator law of cosines", but also provides a somewhat better understanding, with the help of Lemma 1 and properties of the linear invariants discussed in Section 4, how the quadratic invariants act on solutions of the original time-dependent Schrödinger equation. Indeed by (4.20) for two different solutions, say $A_{1}=\mu_{1}$ and $A_{2}=\mu_{2}$, of the characteristic equation, (4.5), we have in an operator form:

$$
\psi(x, t)=K_{1}(t)\left[\chi_{1}(y)\right]=K_{2}(t)\left[\chi_{2}(y)\right]
$$

in view of uniqueness of the Cauchy initial value problem (see, for example, Refs. [8] and [78]). Then by (4.22):

$$
P_{1} \psi=e^{\int_{0}^{t}(c-d) d s} K_{1}\left(y \chi_{1}\right), \quad P_{2} \psi=e^{\int_{0}^{t}(c-d) d s} K_{2}\left(y \chi_{2}\right)
$$

and

$$
\begin{aligned}
E \psi= & e^{-\int_{0}^{t}(c-d) d s}\left[C_{1} P_{1}^{2} \psi+C_{2} P_{2}^{2} \psi+C_{3}\left(P_{1} P_{2}+P_{2} P_{1}\right) \psi\right] \\
= & e^{\int_{0}^{t}(c-d) d s}\left[C_{1} K_{1}\left(y^{2} \chi_{1}\right)+C_{2} K_{2}\left(y^{2} \chi_{2}\right)\right] \\
& +C_{3}\left[P_{1}\left(K_{2}\left(y \chi_{2}\right)\right)+P_{2}\left(K_{1}\left(y \chi_{1}\right)\right)\right]
\end{aligned}
$$


We have

$$
\begin{array}{ll}
K_{2}\left(y \chi_{2}\right)=K_{1}\left(\varphi_{1}\right), & \varphi_{1}=K_{1}^{-1}(0)\left[K_{2}(0)\left(y \chi_{2}\right)\right], \\
K_{1}\left(y \chi_{1}\right)=K_{2}\left(\varphi_{2}\right), & \varphi_{2}=K_{2}^{-1}(0)\left[K_{1}(0)\left(y \chi_{1}\right)\right]
\end{array}
$$

with the help of an analog of the Fourier transform. Therefore

$$
P_{1}\left(K_{2}\left(y \chi_{2}\right)\right)=e^{\int_{0}^{t}(c-d) d s} K_{1}\left(y \varphi_{1}\right), \quad P_{2}\left(K_{1}\left(y \chi_{1}\right)\right)=e^{\int_{0}^{t}(c-d) d s} K_{2}\left(y \varphi_{2}\right),
$$

and finally

$$
E \psi=e^{\int_{0}^{t}(c-d) d s}\left[C_{1} K_{1}\left(y^{2} \chi_{1}\right)+C_{2} K_{2}\left(y^{2} \chi_{2}\right)+C_{3}\left(K_{1}\left(y \varphi_{1}\right)+K_{2}\left(y \varphi_{2}\right)\right)\right],
$$

where each term satisfies the corresponding Schrödinger equation. By the superposition principle we arrive at a new solution in a complete agreement with our Lemma 1. The corresponding initial data follow from (17.4) at $t=0$ and the time-evolution operator (1.10) can be applied (see also Eq. (7.17) for an eigenfunction expansion).

On the second hand one can always expand a square-integrable solution (4.20) of the Cauchy initial value problem in the standard form

$$
\begin{aligned}
\psi(x, t) & =\int_{-\infty}^{\infty} K(x, y, t) \chi(y) d y \\
& =\sum_{n=0}^{\infty} c_{n}(t) \Psi_{n}(x, t)
\end{aligned}
$$

where

$$
c_{n}(t)=\int_{-\infty}^{\infty} \Psi_{n}^{*}(x, t) \psi(x, t) d x
$$

(we use the asterisk for complex conjugate) by the Riesz-Fisher theorem [70], 77, [72] due to completeness of the eigenfunctions (5.34)-(5.36) at all times. Then by the Fubini theorem:

$$
c_{n}(t)=\int_{-\infty}^{\infty} \chi(y)\left(\int_{-\infty}^{\infty} \Psi_{n}^{*}(x, t) K(x, y, t) d x\right) d y
$$

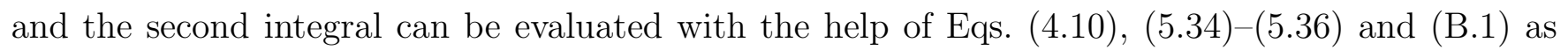
follows

$$
\begin{aligned}
& \int_{-\infty}^{\infty} \Psi_{n}^{*}(x, t) K(x, y, t) d x \\
& =\frac{i^{n} e^{-i(n+1 / 2) \varphi}}{\left(\sqrt{\pi} 2^{n} n !\right)^{1 / 2}}\left(\frac{\sqrt{C_{0}}}{C_{0}\left(\frac{\kappa_{1}}{\kappa}\right)^{2}+\left(\frac{\kappa_{1} \kappa^{\prime}-\kappa_{1}^{\prime} \kappa}{2 a}\right)^{2}}\right)^{1 / 4} \exp \left(\frac{1}{2} \int_{0}^{t}(d-c) d s\right) \\
& \quad \times \exp \left(i\left(\gamma+\frac{\beta^{2}(0) \kappa_{1}^{2}(0)}{4 a \kappa_{1}} \frac{\kappa\left(\kappa_{1} \kappa^{\prime}-\kappa_{1}^{\prime} \kappa\right)}{C_{0}\left(\frac{\kappa_{1}}{\kappa}\right)^{2}+\left(\frac{\kappa_{1} \kappa^{\prime}-\kappa_{1}^{\prime} \kappa}{2 a}\right)^{2}}\right) y^{2}\right)
\end{aligned}
$$




$$
\times \exp \left(-\frac{\beta^{2}(0) \kappa_{1}^{2}(0) \sqrt{C_{0}}}{C_{0}\left(\frac{\kappa_{1}}{\kappa}\right)^{2}+\left(\frac{\kappa_{1} \kappa^{\prime}-\kappa_{1}^{\prime} \kappa}{2 a}\right)^{2}} \frac{y^{2}}{2}\right) H_{n}\left(\frac{\beta(0) \kappa_{1}(0) C_{0}^{1 / 4} y}{\sqrt{C_{0}\left(\frac{\kappa_{1}}{\kappa}\right)^{2}+\left(\frac{\kappa_{1} \kappa^{\prime}-\kappa_{1}^{\prime} \kappa}{2 a}\right)^{2}}}\right) .
$$

Here $\kappa_{1}=\mu \exp \left(\int_{0}^{t}(d-c) d s\right)$ and $\kappa$ are the corresponding solutions of auxiliary equation (5.3) with $C_{0}=0$ and $C_{0} \neq 0$, respectively, with the Wronskian $W\left(\kappa_{1}, \kappa\right)=\kappa_{1} \kappa^{\prime}-\kappa_{1}^{\prime} \kappa$ and

$$
\begin{gathered}
\tan \varphi=\frac{\kappa}{\kappa_{1}} \frac{\kappa_{1} \kappa^{\prime}-\kappa_{1}^{\prime} \kappa}{2 a \sqrt{C_{0}}} \\
C_{0}\left(\frac{\kappa_{1}}{\kappa}\right)^{2}+\left(\frac{\kappa_{1} \kappa^{\prime}-\kappa_{1}^{\prime} \kappa}{2 a}\right)^{2}=\text { constant }
\end{gathered}
$$

by (A.4). (The computational details are left to the reader; our equation (7.10) is equivalent to the classical Ermakov invariant [21].) We may choose $\beta(0) \kappa_{1}(0)=1$ and arrive at the following result.

Theorem 2. (Eigenfunction Expansions) Solution of the Cauchy initial value problem (4.20) in $L^{2}(\mathbb{R})$ can be obtained as an infinite series of multiples of the quadratic invariant eigenfunctions (5.34):

$$
\psi(x, t)=\sum_{n=0}^{\infty} c_{n}(t) \Psi_{n}(x, t),
$$

where the time-dependent coefficients are given by

$$
\begin{aligned}
c_{n}(t)= & i^{n}\left(\frac{\delta}{\sqrt{\pi} 2^{n} n !}\right)^{1 / 2} e^{-i(n+1 / 2) \varphi} \exp \left(\frac{1}{2} \int_{0}^{t}(d-c) d s\right) \\
& \times \int_{-\infty}^{\infty} \exp \left(i \xi y^{2}\right) e^{-\delta^{2} y^{2} / 2} H_{n}(\delta y) \chi(y) d y .
\end{aligned}
$$

Here $\kappa_{1}(t)$ and $\kappa(t)$ are real-valued solutions of the homogeneous and nonhomogeneous auxiliary equations (5.3), respectively, with the Wronskian $W(t)=\kappa_{1} \kappa^{\prime}-\kappa_{1}^{\prime} \kappa$. The phases $\varphi(t)$ and $\gamma(t)$ are determined in terms of these solutions as follows

$$
\begin{gathered}
\varphi=\arctan \left(\frac{\kappa}{\kappa_{1}} \frac{\kappa_{1} \kappa^{\prime}-\kappa_{1}^{\prime} \kappa}{2 a \sqrt{C_{0}}}\right), \quad \frac{d \varphi}{d t}=2 \sqrt{C_{0}} \frac{a}{\kappa^{2}} \\
\frac{d \gamma}{d t}+\frac{a}{\kappa_{1}^{2}}=0
\end{gathered}
$$

and

$$
\begin{gathered}
\delta=\frac{C_{0}^{1 / 4}}{\sqrt{C_{0}\left(\frac{\kappa_{1}}{\kappa}\right)^{2}+\left(\frac{\kappa_{1} \kappa^{\prime}-\kappa_{1}^{\prime} \kappa}{2 a}\right)^{2}}}>0, \\
\xi=\gamma+\frac{\delta^{2}}{2 \sqrt{C_{0}}} \frac{\left(\kappa_{1} \kappa^{\prime}-\kappa_{1}^{\prime} \kappa\right) \kappa}{2 a \kappa_{1}}
\end{gathered}
$$

are constants. A spectral decomposition of the quadratic invariant $E$ in the space of $L^{2}$-solutions is given by

$$
E(t) \psi(x, t)=\omega(t) \sum_{n=0}^{\infty} c_{n}(t)\left(n+\frac{1}{2}\right) \Psi_{n}(x, t)
$$


where the "frequency" $\omega(t)$ is defined by (5.28).

It is worth noting that

$$
\frac{d}{d t}\left[\gamma+\frac{\delta^{2}}{2 \sqrt{C_{0}}} \frac{\kappa}{\kappa_{1}}\left(\frac{W}{2 a}\right)\right]=0
$$

by (7.14) and (A.6), which means that the phase factor (7.16) in front of $y^{2}$ in the second integral of Eq. (7.12) is indeed a constant. The second equation (17.13) follows from (7.18) with the help of (7.9) and (7.14).

Finally by choosing in Eqs. (7.11) -(7.12) a special (square) integrable initial data of the form

$$
\chi_{m}(y)=\exp \left(-i \xi y^{2}\right) e^{-\delta^{2} y^{2} / 2} H_{m}(\delta y)
$$

we conclude in view of the orthogonality property of Hermite polynomials that the time-dependent wave functions

$$
\begin{aligned}
\psi_{m}(x, t)= & D_{m} \exp \left(\frac{1}{2} \int_{0}^{t}(d-c) d s\right) \\
& \times e^{-i(m+1 / 2) \varphi(t)} \Psi_{m}(x, t)
\end{aligned}
$$

are particular solutions of the Schrödinger equation (1.1)-(1.2) for arbitrary constants $D_{m}$ :

$$
i \frac{\partial \psi_{m}}{\partial t}=H \psi_{m}, \quad E \psi_{m}=\omega\left(m+\frac{1}{2}\right) \psi_{m} .
$$

They are also eigenfunctions of the quadratic invariant $E$ corresponding to the discrete "spectrum":

$$
\langle E\rangle_{m}=\int_{-\infty}^{\infty} \psi_{m}^{*} E \psi_{m} d x=\left|D_{m}\right|^{2} \omega_{0}\left(m+\frac{1}{2}\right),
$$

see (5.33). (It can be verified by direct substitution; the details are left to the reader.) The explicit wave functions (7.20) are derived here without separation of the variables with the aid of our Theorem 2 and certain variants of the Ermakov invariant. If $\left|D_{m}\right|=1$, solution (7.11) takes the form

$$
\psi(x, t)=\sum_{n=0}^{\infty} i^{n} e^{-i(n+1 / 2) \varphi(0)} \psi_{n}(x, t) \int_{-\infty}^{\infty} \psi_{n}^{*}(y, 0) \chi(y) d y
$$

in terms of the time-dependent wave functions (7.20) provided that

$$
\varepsilon(0)=\frac{C_{0}^{1 / 4}}{\kappa(0)}=\delta, \quad \frac{(c(0)+d(0)) \kappa(0)-\kappa^{\prime}(0)}{4 a(0) \kappa(0)}=\xi
$$

(see also [85] for the path-integral method). The traditional operator approach (for the parametric oscillator) is presented in [48] and/or elsewhere (see also [1] and [39]).

\section{A General Nonlinear Superposition Principle for Ermakov's Equations}

The Pinney superposition formula (6.8)-(6.9) allows one to construct solutions of the nonlinear auxiliary equation (5.3) in terms of given solutions of the corresponding linear equation. In general 
we take two different solutions, say $\kappa_{1}$ and $\kappa_{2}$, of the generalized Ermakov equations (5.3) with $C_{0}^{(1,2)} \neq 0$ and consider two quadratic invariants:

$$
\begin{aligned}
& E_{1}(t)=\left[\left(\kappa_{1} p+\frac{(c+d) \kappa_{1}-\kappa_{1}^{\prime}}{2 a} x\right)^{2}+\frac{C_{0}^{(1)}}{\kappa_{1}^{2}} x^{2}\right] \lambda(t), \\
& E_{2}(t)=\left[\left(\kappa_{2} p+\frac{(c+d) \kappa_{2}-\kappa_{2}^{\prime}}{2 a} x\right)^{2}+\frac{C_{0}^{(2)}}{\kappa_{2}^{2}} x^{2}\right] \lambda(t) .
\end{aligned}
$$

Their arbitrary linear combination,

$$
D_{1} E_{1}(t)+D_{2} E_{2}(t)=E(t)
$$

( $D_{1}$ and $D_{2}$ are constants), is also a quadratic invariant given by (5.2) for a certain solution $\kappa$ of the nonlinear auxiliary equation (5.3). Thus the following operator identity holds

$$
\begin{aligned}
(\kappa p & \left.+\frac{(c+d) \kappa-\kappa^{\prime}}{2 a} x\right)^{2}+\frac{C_{0}}{\kappa^{2}} x^{2} \\
= & D_{1}\left[\left(\kappa_{1} p+\frac{(c+d) \kappa_{1}-\kappa_{1}^{\prime}}{2 a} x\right)^{2}+\frac{C_{0}^{(1)}}{\kappa_{1}^{2}} x^{2}\right] \\
& +D_{2}\left[\left(\kappa_{2} p+\frac{(c+d) \kappa_{2}-\kappa_{2}^{\prime}}{2 a} x\right)^{2}+\frac{C_{0}^{(2)}}{\kappa_{2}^{2}} x^{2}\right]
\end{aligned}
$$

and in a similar fashion we arrive at a general nonlinear superposition principle for the solutions of Ermakov's equations (5.3):

$$
\kappa^{2}(t)=D_{1} \kappa_{1}^{2}(t)+D_{2} \kappa_{2}^{2}(t)
$$

where the constant $C_{0}$ is given by

$$
C_{0}-C_{0}^{(1)} D_{1}^{2}-C_{0}^{(2)} D_{2}^{2}=D_{1} D_{2}\left[\frac{W^{2}\left(\kappa_{1}, \kappa_{2}\right)}{(2 a)^{2}}+C_{0}^{(1)} \frac{\kappa_{2}^{2}}{\kappa_{1}^{2}}+C_{0}^{(2)} \frac{\kappa_{1}^{2}}{\kappa_{2}^{2}}\right]
$$

with $W\left(\kappa_{1}, \kappa_{2}\right)$ being the Wronskian of two solutions (see Appendix A). One can also derive this property by adding the corresponding solutions (5.7)-(5.9) of the original linear system (5.4) - (5.6) or with the help of the Pinney formula (6.8)-(6.9). The details are left to the reader.

Acknowledgments. The author thanks George E. Andrews, Carlos Castillo-Chávez, Victor V. Dodonov, Vladimir I. Man'ko, Svetlana Roudenko and Kurt Bernardo Wolf for support, valuable comments and encouragement. I am grateful to Peter G. L. Leach for careful reading of the manuscript - his numerous suggestions have helped to improve the presentation.

\section{Appendix A. Required Transformations}

Equating coefficients in front of the operators $p^{2}, x^{2}$ and $p x+x p$ in (6.4) $-(\underline{6.5})$ with the help of (6.7) one gets

$$
\begin{array}{r}
\kappa^{2}=C_{1} \kappa_{1}^{2}+C_{2} \kappa_{2}^{2}+2 C_{3} \kappa_{1} \kappa_{2}, \\
{\left[\frac{(c+d) \kappa-\kappa^{\prime}}{2 a}\right]^{2}+\frac{C_{0}}{\kappa^{2}}}
\end{array}
$$




$$
\begin{aligned}
= & C_{1}\left[\frac{(c+d) \kappa_{1}-\kappa_{1}^{\prime}}{2 a}\right]^{2}+C_{2}\left[\frac{(c+d) \kappa_{2}-\kappa_{2}^{\prime}}{2 a}\right]^{2} \\
& +2 C_{3} \frac{\left[(c+d) \kappa_{1}-\kappa_{1}^{\prime}\right]\left[(c+d) \kappa_{2}-\kappa_{2}^{\prime}\right]}{(2 a)^{2}}, \\
\kappa & \frac{(c+d) \kappa-\kappa^{\prime}}{2 a} \\
= & C_{1} \kappa_{1} \frac{(c+d) \kappa_{1}-\kappa_{1}^{\prime}}{2 a}+C_{2} \kappa_{2} \frac{(c+d) \kappa_{2}-\kappa_{2}^{\prime}}{2 a} \\
& +C_{3}\left[\kappa_{1} \frac{(c+d) \kappa_{2}-\kappa_{2}^{\prime}}{2 a}+\kappa_{2} \frac{(c+d) \kappa_{1}-\kappa_{1}^{\prime}}{2 a}\right],
\end{aligned}
$$

respectively. Multiply (A.2) by (A.1) and use (A.3) in the left hand side in order to obtain (6.9) as a result of elementary but rather tedious calculations.

A general nonlinear superposition principle for Ermakov's equations, given by (8.5) -(8.6), is derived from the quadratic invariant identity (8.4) in a similar fashion. In addition the constant in the right hand side of (8.6) (see also (17.10) ) can be verified by direct differentiation as follows

$$
\begin{aligned}
\frac{d}{d t} & {\left[\left(\frac{W}{2 a}\right)^{2}+C_{0}^{(1)}\left(\frac{\kappa_{2}}{\kappa_{1}}\right)^{2}+C_{0}^{(2)}\left(\frac{\kappa_{1}}{\kappa_{2}}\right)^{2}\right] } \\
= & \frac{2 W}{(2 a)^{2}}\left(W^{\prime}-\frac{a^{\prime}}{a} W\right)+2 W\left(C_{0}^{(1)} \frac{\kappa_{2}}{\kappa_{1}^{3}}-C_{0}^{(2)} \frac{\kappa_{1}}{\kappa_{2}^{3}}\right) \\
= & \frac{2 W}{(2 a)^{2}}\left(W^{\prime}-\frac{a^{\prime}}{a} W\right) \\
& -\frac{2 W}{(2 a)^{2}}\left[\left(\kappa_{1} \kappa_{2}^{\prime}-\kappa_{1}^{\prime} \kappa_{2}\right)^{\prime}-\frac{a^{\prime}}{a}\left(\kappa_{1} \kappa_{2}^{\prime}-\kappa_{1}^{\prime} \kappa_{2}\right)\right]=0
\end{aligned}
$$

with the help of auxiliary equations (5.3). (It is a natural generalization of the classical Ermakov invariant [21].) En route, we derive an identity:

$$
\frac{1}{2 a}\left(\frac{W}{2 a}\right)^{\prime}+C_{0}^{(1)} \frac{\kappa_{2}}{\kappa_{1}^{3}}-C_{0}^{(2)} \frac{\kappa_{1}}{\kappa_{2}^{3}}=0,
$$

which may be considered as an extension of the familiar Abel theorem, occurring when $C_{0}^{(1)}=$ $C_{0}^{(2)}=0$, to the nonlinear Ermakov equations. Another identity,

$$
\begin{aligned}
\frac{d}{d t} & {\left[\frac{\kappa_{2}}{\kappa_{1}}\left(\frac{W}{2 a}\right)\right] } \\
& =\frac{2 a}{\kappa_{1}^{2}}\left[\left(\frac{W}{2 a}\right)^{2}-C_{0}^{(1)}\left(\frac{\kappa_{2}}{\kappa_{1}}\right)^{2}+C_{0}^{(2)}\left(\frac{\kappa_{1}}{\kappa_{2}}\right)^{2}\right],
\end{aligned}
$$

has been useful in Section 7 with $C_{0}^{(1)}=0, C_{0}^{(2)}=C_{0}$ and $\kappa_{2}=\kappa$ when the right hand side reduces to a multiple of the Ermakov invariant (see Eq. (7.18) ). 


\section{Appendix B. An Integral Evaluation}

In Section 7 (see Eq. (7.8)) we use the following integral

$$
\begin{aligned}
& \int_{-\infty}^{\infty} e^{-\lambda^{2}(x-y)^{2}} H_{n}(a y) d y \\
& \quad=\frac{\sqrt{\pi}}{\lambda^{n+1}}\left(\lambda^{2}-a^{2}\right)^{n / 2} H_{n}\left(\frac{\lambda a x}{\left(\lambda^{2}-a^{2}\right)^{1 / 2}}\right), \quad \operatorname{Re} \lambda^{2}>0,
\end{aligned}
$$

which is equivalent to Eq. (30) on page 195 of Vol. 2 of Ref. [19] (the Gauss transform of Hermite polynomials), or Eq. (17) on page 290 of Vol. 2 of Ref. [20].

\section{REFERENCES}

[1] K. Andriopoulos and P. G. L. Leach, Wavefunctions for the time-dependent linear oscillator and Lie point symmetries, J. Phys. A 38 (2005) \#20, 4365-4374.

[2] M. V. Berry, Classical adiabatic angles and quantum adiabatic phase, J. Phys. A: Math. Gen. 18 (1985) \# 1, $15-27$.

[3] M. V. Berry and J. Hannay, Classical non-adiabatic angles, J. Phys. A: Math. Gen. 21 (1988) \# 6, L325-L331.

[4] C. Bertoni, F. Finelli and G. Venturi, Adiabatic invariants and scalar fields in a de Sitter space-time, Phys. Lett. A 237 (1998), 331-336.

[5] N. N. Bogoliubov and D. V. Shirkov, Introduction to the Theory of Quantized Fields, third edition, John Wiley \& Sons, New York, Chichester, Brisbane, Toronto, 1980.

[6] R. Cordero-Soto, R. M. Lopez, E. Suazo and S. K. Suslov, Propagator of a charged particle with a spin in uniform magnetic and perpendicular electric fields, Lett. Math. Phys. 84 (2008) \#2-3, 159-178.

[7] R. Cordero-Soto, E. Suazo and S. K. Suslov, Models of damped oscillators in quantum mechanics, Journal of Physical Mathematics, 1 (2009), S090603 (16 pages).

[8] R. Cordero-Soto, E. Suazo and S. K. Suslov, Quantum integrals of motion for variable quadratic Hamiltonians, Annals of Physics, to appear; see also arXiv:0912.4900v8 [math-ph] 31 Jan 2010.

[9] R. Cordero-Soto and S. K. Suslov, Time reversal for modified oscillators, Theoretical and Mathematical Physics 162 (2010) \#3, 286-316; see also arXiv:0808.3149v9 [math-ph] 8 Mar 2009.

[10] E. D. Courant and H. S. Snyder, Theory of the alternating-gradient synchrotron, Ann. Phys. (N. Y.) 3 (1958), $1-48$.

[11] F. Dalfovo, S. Giorgini, L. P. Pitaevskii and S. Stringari, Theory of Bose-Einstein condensation in trapped gases, Rev. Mod. Phys. 71 (1999), 463-512.

[12] L. Debnath and P. Mikusiński, Hilbert Spaces with Applications, third edition, Elsevier Academic Press, Amsterdam, Boston, etc., 2005.

[13] H. Dekker, Classical and quantum mechanics of the damped harmonic oscillator, Phys. Rep. 80 (1981), 1-112.

[14] V. V. Dodonov, I. A. Malkin and V. I. Man'ko, Integrals of motion, Green functions, and coherent states of dynamical systems, Int. J. Theor. Phys. 14 (1975) \# 1, 37-54.

[15] V. V. Dodonov and V. I. Man'ko, Invariants and correlated states of nonstationary quantum systems, in: Invariants and the Evolution of Nonstationary Quantum Systems, Proceedings of Lebedev Physics Institute, vol. 183, pp. 71-181, Nauka, Moscow, 1987 [in Russian]; English translation published by Nova Science, Commack, New York, 1989, pp. 103-261.

[16] E. V. Doktorov, I. A. Malkin and V. I. Man'ko, Dynamical symmetry of vibronic transitions in polyatomic molecules and Frank-Condon principle, J. Mol. Spectrosc. 64 (1977), 302-326.

[17] P. Ehrenfest, Bemerkung über die angenäherte Gültigkeit der klassischen Mechanik innerhalb der Quantenmechanik, Zeitschrift für Physik A 45 (1927), 455-457.

[18] C. J. Eliezer and A. Grey, A note on the time-dependent harmonic oscillator, SIAM J. Appl. Math. 30 (1976) \#3, $463-468$.

[19] A. Erdélyi, Higher Transcendental Functions, Vols. I-III, A. Erdélyi, ed., McGraw-Hill, 1953.

[20] A. Erdélyi, Tables of Integral Transforms, Vols. I-II, A. Erdélyi, ed., McGraw-Hill, 1954. 
[21] V. P. Ermakov, Second-order differential equations. Conditions of complete integrability, Universita Izvestia Kiev, Series III 9 (1880), 1-25; see also Appl. Anal. Discrete Math. 2 (2008) \#2, 123-145 for English translation of Ermakov's original paper.

[22] L. D. Faddeev, Feynman integrals for singular Lagrangians, Theoretical and Mathematical Physics 1 (1969) \#1, 3-18 [in Russian].

[23] R. P. Feynman, The Principle of Least Action in Quantum Mechanics, Ph. D. thesis, Princeton University, 1942; reprinted in: "Feynman's Thesis - A New Approach to Quantum Theory", (L. M. Brown, Editor), World Scientific Publishers, Singapore, 2005, pp. 1-69.

[24] R. P. Feynman, Space-time approach to non-relativistic quantum mechanics, Rev. Mod. Phys. 20 (1948) \# 2, 367-387; reprinted in: "Feynman's Thesis - A New Approach to Quantum Theory", (L. M. Brown, Editor), World Scientific Publishers, Singapore, 2005, pp. 71-112.

[25] R. P. Feynman and A. R. Hibbs, Quantum Mechanics and Path Integrals, McGraw-Hill, New York, 1965.

[26] F. Finelli, A. Gruppuso and G. Venturi, Quantum fields in an expanding universe, Class. Quantum Grav. 16 (1999), 3923-3935.

[27] F. Finelli, G. P. Vacca and G. Venturi, Chaotic inflation from a scalar field in nonclassical states, Phys. Rev. D 58 (1998), 103514 (14 pages).

[28] J. H. Hannay, Angle variable holonomy in adiabatic excursion of an integrable Hamiltonian, J. Phys. A: Math. Gen 18 (1985) \# 2, 221-230.

[29] R. M. Hawkins and J. E. Lidsey, Ermakov-Pinney equation in scalar field cosmologies, Phys. Rev. D 66 (2002), 023523 (8 pages).

[30] Yu. Kagan, E. L. Surkov and G. V. Shlyapnikov, Evolution of Bose-condensed gas under variations of the confining potential, Phys. Rev. A 54 (1996) \#3, R1753-R1756.

[31] Yu. Kagan, E. L. Surkov and G. V. Shlyapnikov, Evolution of Bose gas in anisotropic time-dependent traps, Phys. Rev. A 55 (1997) \#1, R18-R21.

[32] Yu. S. Kivshar, T. J. Alexander and S. K. Turitsyn, Nonlinear modes of a macroscopic quantum oscillator, Phys. Lett. A 278 (2001) \#1, 225-230.

[33] J. R. Klauder and E. C. G. Sudarshan, Fundamentals of Quantum Optics, Benjamin, New York, 1968.

[34] D. Kochan, Quantization of equations of motion, Acta Polytechnica 47 (2007) \#2-3, 60-67.

[35] D. Kochan, Functional integral for non-Lagrangian systems, Phys. Rev. A 81 (2010) \#2, 022112.

[36] M. Kruskal, Asymptotic theory of Hamiltonian and other systems with all solutions nearly periodic, J. Math. Phys. 3 (1962), 806-828.

[37] L. D. Landau and E. M. Lifshitz, Quantum Mechanics: Nonrelativistic Theory, Pergamon Press, Oxford, 1977.

[38] N. Lanfear and S. K. Suslov, The time-dependent Schrödinger equation, Riccati equation and Airy functions, arXiv:0903.3608v5 [math-ph] 22 Apr 2009.

[39] P. G. L. Leach, Berry's phase and wave functions for time-dependent Hamiltonian systems, J. Phys. A: Math. Gen 23 (1990), 2695-2699.

[40] P. G. L. Leach and K. Andriopoulos, The Ermakov equation: a commentary, Appl. Anal. Discrete Math. 2 (2008) \#2, 146-157.

[41] P. G. L. Leach and K. Andriopoulos, An invariant for the doubly generalized classical Ermakov-Pinney system and its quantal equivalent, Phys. Scripta 77 (2008), 015002 (7 pages).

[42] P. G. L. Leach, A. Karasu (Kalkanli), M. C. Nucci and K. Andriopoulos, Ermakov's superintegrable toy and nonlocal symmetries, Symmetry, Integrability and Geometry: Methods and Applications, SIGMA 1 (2005), 018 (15 pages).

[43] H. R. Lewis, Jr., Classical and quantum systems with time-dependent harmonic-oscillator-type Hamiltonians, Phys. Rev. Lett. 18 (1967) \#13, 510-512.

[44] H. R. Lewis, Jr., Motion of a time-dependent harmonic oscillator, and of a charged particle in a class of timedependent, axially symmetric electromagnetic fields, Phys. Rev. 172 (1968) \#5, 1313-1315.

[45] H. R. Lewis, Jr., Class of exact invariants for classical and quantum time-dependent harmonic oscillators, J. Math. Phys. 9 (1968) \#11, 1976-1986.

[46] H. R. Lewis and P. G. L. Leach, Exact invariants for a class of time-dependent nonlinear Hamiltonian systems, J. Math. Phys. 23 (1982) \#1, 165-175.

[47] H. R. Lewis and P. G. L. Leach, A direct approach to finding exact invariants for one-diminsional time-dependent classical Hamiltonians, J. Math. Phys. 23 (1982) \#12, 2371-2374. 
[48] H. R. Lewis, Jr., and W. B. Riesenfeld, An exact quantum theory of the time-dependent harmonic oscillator and of a charged particle in a time-dependent electromagnetic field, J. Math. Phys. 10 (1969) \#8, 1458-1473.

[49] E. H. Lieb and M. Loss, Analysis, second edition, Grad. Stud. Math. 14, AMS, Providence, RI, 2001.

[50] R. M. Lopez and S. K. Suslov, The Cauchy problem for a forced harmonic oscillator, Revista Mexicana de Física, 55 (2009) \#2, 195-215; see also arXiv:0707.1902v8 [math-ph] 27 Dec 2007.

[51] A. Maharaj and P. G. L. Leach, The method of reduction of order and linearization of the two-dimensional Ermakov system, Math. Meth. in Appl. Sciences 30 (2007) \#16, 2125-2145.

[52] F. G. Major, V. N. Gheorghe and G. Werth, Charged Particle Traps, Springer-Verlag, Berlin, Heidelberg, 2005.

[53] I. A. Malkin and V. I. Man'ko, Dynamical Symmetries and Coherent States of Quantum System, Nauka, Moscow, 1979 [in Russian].

[54] I. A. Malkin, V. I. Man'ko and D. A. Trifonov, Invariants and the evolution of coherent states for a charged particle in a time-dependent magnetic field, Phys. Lett. A. 30 (1969) \#7, 414.

[55] I. A. Malkin, V. I. Man'ko and D. A. Trifonov, Coherent states and transition probabilities in a time-dependent electromagnetic field, Phys. Rev. D. 2 (1970) \#2, 1371-1385.

[56] I. A. Malkin, V. I. Man'ko and D. A. Trifonov, Linear adiabatic invariants and coherent states, J. Math. Phys. 14 (1973) \#5, 576-582.

[57] A. Mandilara, E. Karpov and N. J. Cerf, Non-Gaussianity bounded uncertainty relation for mixed states, arXiv:0910.3474v1 [quant-ph] 19 Oct 2009.

[58] V. P. Maslov and M. V. Fedoriuk, Semiclassical Approximation in Quantum Mechanics, Reidel, Dordrecht, Boston, 1981.

[59] M. Meiler, R. Cordero-Soto and S. K. Suslov, Solution of the Cauchy problem for a time-dependent Schrödinger equation, J. Math. Phys. 49 (2008) \#7, 072102: 1-27; published on line 9 July 2008, URL: http://link.aip.org/link/?JMP/49/072102 see also arXiv: 0711.0559v4 [math-ph] 5 Dec 2007.

[60] E. Merzbacher, Quantum Mechanics, third edition, John Wiley \& Sons, New York, 1998.

[61] W. E. Milne, The numerical determination of characteristic numbers, Phys. Rev. 35 (1930) \#7, 863-867.

[62] D. A. Morales, Correspondence betweem Berry's phase and Lewis's phase for quadratic Hamiltonians, J. Phys. A: Math. Gen 21 (1988) \# 18, L889-L892.

[63] C. A. Muñoz, J. Rueda-Paz and K. B. Wolf, Discrete repulsive oscillator wavefunctions, J. Phys. A: Math. Theor. 42 (2009), 485210 (12pp).

[64] A. F. Nikiforov, S. K. Suslov and V. B. Uvarov, Classical Orthogonal Polynomials of a Discrete Variable, Springer-Verlag, Berlin, New York, 1991.

[65] M. C. Nucci and P. G. L. Leach, Jacobi's last multiplier and the complete symmetry group of the Ermakov-Pinney equation, J. Nonlinear Math. Phys. 12 (2005) \#2, 305-320.

[66] Y-G. Oh, Cauchy problem and Ehrenfest's law of nonlinear Schrödinger equations with potentials, J. Differential Equations 81 (1989), 255-274.

[67] P. B. E. Padilla, Ermakov-Lewis dynamic invariants with some applications, arXiv:0002005v3 [math-ph] 18 Mar 2000.

[68] V. M. Pérez-García, P. Torres and G. D. Montesinos, The method of moments for nonlinear Schrödinger equations: theory and applications, SIAM J. Appl. Math. 67 (2007) \#4, 990-1015.

[69] E. Pinney, The nonlinear differential equation $y^{\prime \prime}+p(x) y+c y^{-3}=0$, Proc. Am. Math. Soc. 1 (1950), 681.

[70] M. Reed and B. Simon, Methods of Modern Mathematical Physics I: Functional Analysis, Academic Press, New York, San Francisco, London, 1972.

[71] M. Reed and B. Simon, Methods of Modern Mathematical Physics II: Fourier Analysis and Self-Adjointness, Academic Press, New York, Boston, London, 1975.

[72] W. Rudin, Principles of Mathematical Analysis, third edition, McGraw-Hill, New York, 1976.

[73] L. I. Schiff, Quantum Mechanics, third edition, McGraw-Hill, New York, 1968.

[74] D. Schuch, Riccati and Ermakov equations in time-dependent and time-independent quantum systems, Symmetry, Integrability and Geometry: Methods and Applications, SIGMA 4 (2008), 043 (16 pages).

[75] Yu. F. Smirnov and K. V. Shitikova, The method of K harmonics and the shell model, Soviet Journal of Particles \& Nuclei 8 (1977) \#4, 344-370.

[76] E. Suazo, On Schrödinger equation with tine-dependent quadratic Hamiltonians in $\mathbb{R}^{d}$, arXiv:0912.2113v2 [mathph] 11 Dec 2009. 
[77] E. Suazo and S. K. Suslov, An integral form of the nonlinear Schrödinger equation with wariable coefficients, arXiv:0805.0633v2 [math-ph] 19 May 2008.

[78] E. Suazo and S. K. Suslov, Cauchy problem for Schrödinger equation with variable quadratic Hamiltonians, under preparation.

[79] E. Suazo, S. K. Suslov and J. M. Vega, The Riccati differential equation and a diffusion-type equation, arXiv:0807.4349v4 [math-ph] 8 Aug 2008.

[80] K. R. Symon, The adiabatic invariant of the linear and nonlinear oscillator, J. Math. Phys. 11 (1970) \#4, $1320-1330$.

[81] V. E. Tarasov, Quantization of non-Hamiltonian and dissipative systems, Phys. Lett. A 288 (2001), 173-182.

[82] Ch-I. Um, K-H. Yeon and T. F. George, The quantum damped oscillator, Phys. Rep. 362 (2002), 63-192.

[83] G. Velo, Mathematical aspects of the nonlinear Schrödinger equation, in: Nonlinear Klein-Gordon and Schrödinger Systems: Theory and Applications (L. Vázquez, L. Streit, and V. P. Pérez-Garsia, Editors), World Scientific, Singapore, 1996, pp. 39-67.

[84] K. B. Wolf, On time-dependent quadratic Hamiltonians, SIAM J. Appl. Math. 40 (1981) \#3, 419-431.

[85] K-H. Yeon, K-K. Lee, Ch-I. Um, T. F. George and L. N. Pandey, Exact quantum theory of a time-dependent bound Hamiltonian systems, Phys. Rev. A 48 (1993) \# 4, 2716-2720.

School of Mathematical and Statistical Sciences \& Mathematical, Computational and Modeling Sciences Center, Arizona State University, Tempe, AZ 85287-1804, U.S.A.

E-mail address: sks@asu.edu

URL: http://hahn.la.asu.edu/〜 suslov/index.html 